\title{
Le réalisme magique dans les récits d'Ook Chung
}

\author{
Simone Grossman
}

Université Bar Ilan

La présente étude se propose d'examiner la fonction du réalisme magique dans l'œuvre d'Ook Chung. Écrivain québécois, né au Japon de parents coréens et venu très jeune au Québec, Chung a pris conscience de sa différence à l'adolescence (Bernier, 2002, p. 203-206). Plus tard, la création littéraire lui donne les moyens de surmonter les difficultés de sonidentité mêlée, en particulier le réalisme magique, qui résout les conflits de personnages déchirés entre des cultures opposées, comme le montre l'étude de récits tirés de Nouvelles orientales et désorientées (1999 [1994]) et de Contes butô (2003). 


\section{Réalisme magique et postcolonialisme}

Le réalisme magique est une stratégie narrative visant à faire coexister, à l'intérieur d'un récit, une vision rationnelle de la réalité avec le surnaturel. Comme l'expose Amaryll Chanady (1985), les histoires réalistes magiques s'intègrent à un contexte social, historique et politique réel cependant que l'auteur introduit des éléments extraordinaires, mais non signalés comme tels. Chanady montre que les perspectives contraires mises en présence relèvent de systèmes culturels opposés. Dans l'œuvre de Chung cohabitent la pensée occidentale empirique et la mentalité magique.

Le réalisme magique a été pratiqué par les auteurs latinoaméricains et antillais du XX $\mathrm{X}^{\mathrm{e}}$ siècle représentant les tensions entre la mentalité primitive et le rationalisme européen. On le trouve également dans la culture postcoloniale, dont font partie les fictions dont les auteurs sont originaires de pays anciennement colonisés. Selon Stephen Slemon, les narrations réalistes magiques résument les oppositions contenues dans la culture postcolonialiste. Les «voix perdues» et autres « débris » laissés pour compte par les structures impérialistes sont récupérés par le réalisme magique, en réaction à la fragmentation et à la discontinuité causées par le colonialisme (1988, p. 16 et 21). D’après Chanady, le réalisme magique «depicts and usually condemns colonialism ${ }^{1}$ » (2003, p. 32). Marie Vautier range le réalisme magique au nombre des «techniques littéraires postcoloniales des pays du nouveau monde» autorisant «l'acte politique de "write back to the

\footnotetext{
1 « décrit et condamne habituellement le colonialisme », trad. Amy J. Ransom.
} 
center 2" " (Vautier, 2003) et visant à " démanteler, démystifier et démasquer l'autorité culturelle européenne, [...] tout en créant une identité littéraire indépendante" (Tiffin, 1988, p. 171; cit. et trad. par Vautier, 2003).

La littérature du Québec diffère de celle des pays du Nouveau Monde anciennement colonisé en ceci que les colons venus de France ont eux-mêmes été colonisés par les Anglais. Comme l'expose Vautier, les lettres québécoises ont dépassé le stade de la mise en question du rapport au centre impérialiste et ont évolué dans le sens d'un «postcolonialisme de consensus » (Vautier, 2003). Vautier cite à l'appui de sa thèse des romans québécois où la supériorité mythique du colonisateur blanc ou européen est contestée par les personnages amérindiens et métis.

Dans la critique québécoise de la fin du $\mathrm{XX}^{\text {e }}$ siècle, les débats théoriques sur le postcolonialisme ont fait place à des interrogations sur la transculture, l'identitaire et l'écriture migrante (Sugars, 2002, p. 35). Chanady observe que l'approche critique québécoise substitue aux oppositions binaires (colonisateur/colonisé, Blanc/non-Blanc, civilisé/barbare, et autres) posées par Slemon des modèles hybrides plus adaptés aux écrits des immigrants et des écrivains appartenant aux minorités culturelles ${ }^{3}$. Pour Pierre Nepveu, l'imaginaire

\footnotetext{
2 « recentrer par l'écriture » (Vautier, 2003).

3 «And have more recent Quebecois critics not developed more nuanced approaches to their literature that reject binary paradigms of colonizers and colonized in favor of models of hybridity such as "écriture migrante" that are more relevant to contemporary writing by immigrants and cultural minorities and can also be applied to the presence of "otherness" in earlier Quebecois literature? » «Les critiques québécois n'ont-ils pas développé récemment des approches plus nuancées, écartant les paradigmes binaires des colonisateurs et des colonisés en faveur de modèles de l'hybridité, tels que l'“écriture
} 
québécois «s'est largement défini, depuis les années soixante, sous le signe de l'exil», en «imaginaire migrant, pluriel, souvent cosmopolite » (1988, p. 199).

Chung adopte « la perspective du colonisé » dans sa mise en fiction du " rapport difficile à l'origine", comme le fait valoir Gilles Dupuis (2005, p. 108). La quête d'identité de personnages métis, eurasiens ou autres, est également le thème dominant de Nouvelles orientales et désorientées. Nous verrons que le réalisme magique ouvre "une spatialité duelle » apte à faire éclater les «restrictions de l'espace colonial » et à dégager l'espace hybride constitutif d'identité, selon Suzanne Baker (1993, p. 87)4. Dans Contes butô, on trouve les éléments propres au récit réaliste magique, tel que le décrit Vautier, où « l'intrigue se perd dans des contes magiques, mythiques, allégoriques hors temps, hors "réalité" - et [...] pourtant bien ancrés dans l'Histoire » (Vautier, 1991, p. 2).

Notre corpus comprend cinq nouvelles. «La petite princesse et le labyrinthe des rêves » (1999a, p. 27-37), «Le catcher du métro» (1999b, p. 57-68) et «Le royaume silencieux » (1999c, p. 143-154) ont pour décor l'Inde, le Japon et la Chine modernes. « Le baiser de minuit» (2003a), p. 25-39) et «L'enfantement» (2003b, p. 137-145) se déroulent respectivement en Islande et en Corée. Les protagonistes des récits, des personnes déplacées, immigrés ou indigènes de pays

migrante", plus aptes à définir les écrits contemporains des immigrants et des minorités culturelles, et pouvant aussi s'appliquer à la présence de l'“altérité" dans la littérature québécoise plus ancienne? »(Chanady, 2003, p. 32). Je traduis.

4 « magic realism contests the restrictions of colonial space by opening up a “dual spatiality" » (Baker,1993, p. 87). Je traduis. 
du tiers monde anciennement colonisés, sont le plus souvent d'anciens dominés.

Notre étude développera une triple axiomatique relative aux divers aspects du réalisme magique présents dans l'œuvre de Chung. Dans un premier stade, dans "Le catcher du métro », nous verrons que le réalisme magique catalyse l'interpénétration critique des cultures. Dans une deuxième partie, nous examinerons le topos réaliste magique de la lecture et de la bibliothèque régissant l'action dans «La petite princesse et le labyrinthe des rêves ", " Le royaume silencieux » et «Le baiser de minuit ». Le troisième volet de notre étude traitera des personnages d'enfants qui, par-delà leurs dispositions extraordinaires, métaphorisent les colonisés, en particulier dans « L'enfantement».

\section{Réalisme magique et hybridité culturelle}

Dans « Le catcher du métro », le métro de Tokyo, où s'affrontent le Japon traditionnel et la culture occidentale, les travailleurs de nuit, devenus fous, se suicident. Des barrières isolent les voies et des psychiatres surveillent les voyageurs, secondés par des catchers (p. 58) ou attrapeurs, dont fait partie le narrateur, un Japonais qui travaille la nuit. Sa femme, Mithra, une Américaine au prénom oriental, travaille à l'ambassade des États-Unis. Le couple a des triplés. Le catcher ne voit jamais sa famille, car il travaille la nuit et dort le jour. Un soir, une désespérée manque de l'entraîner sous la rame. Renvoyé par ses supérieurs et chassé par sa femme, il aperçoit sa famille en compagnie d'un ancien collègue et ne songe plus qu'à se suicider. 
Le réalisme magique transforme le métro, moyen de transport banal, en enfer chtonien. L'accent est mis sur le gigantisme du réseau de "Tôkyo Metropolis » desservant de lointaines destinations, "de Nakano à Nishi-Funabashi, de Ebisu à Kita-Senju, de Ojikibo à Ikebukuro » (1999b, p. 57), noms de lieux dont l'authenticité contribue à ancrer le récit dans la réalité urbaine. Les interminables trajets quotidiens se muent en nomadisme pour les voyageurs qui perdent leurs repères dans une réalité bien connue d'eux, mais pervertie. En effet, le métro est devenu un monstre qui «déploie ses tentacules dans le ventre de la terre» et dévore les voyageurs ou « lemmings humains » (p. 57-58), oxymoron emphatisant le statut inférieur des «nocturnes», êtres monstrueux mihumains, mi-animaux, privés de soleil, source d'énergie spirituelle. Là où « le bleu des vagues est remplacé par le noir du gouffre " (p. 60) s'opèrent la métamorphose des hommes et également celle des choses, telle la chaise de surveillant de plage transformée en siège d'attrapeur.

Dans la personne du catcher, le réalisme magique produit le cumul de l'ancienne culture du Japon et la nouvelle religion de l'Argent, d'origine étrangère bien sûr. L'Orient et l'Occident sont métaphorisés respectivement par le passé mythique et la vie moderne. Le catcher, écartelé entre son attachement au Japon traditionnel et son amour des voyages, a opté pour le travail de nuit, mieux rémunéré. Il est déchiré entre son passé oriental et le présent où, «à l'ère du village global» (p. 61), l'influence de l'Occident est la plus forte. Dans le métro, lieu de contact entre les civilisations et les mondes, il a rencontré Mithra, une Américaine dont le nom perse est une allusion au culte de Mithra, rival du christianisme naissant, célébrant la renaissance du soleil le jour de Noël. Mithra lui rappelle ses 
voyages du passé, dans le monde entier, en tant que « conseiller technique pour les réseaux métropolitains» (p.61). Dans le métro, lieu cosmopolite, le catcher se sent chez lui bien qu'il exècre le diktat venu de l'étranger, qui l'oblige à se déguiser en père Noël le soir du 24 décembre. Pris comme en étau entre les religions et les mythes, il incarne le choc des cultures et le syncrétisme des religions.

Le métissage culturel du Japon moderne est signifié à plusieurs niveaux sémantiques, d'abord dans la cumulation des traditions culinaires, marquée par la consommation de dinde rôtie accompagnée de saké au réveillon de Noël. La mentalité du catcher le rattache au Japon ancestral. Forcé de dormir dans un "hôtel-capsule» par sa femme qui lui refuse l'accès de sa propre maison, il regrette les «cloisons en $\operatorname{shoji}^{5}$ » (p.66) de l'architecture japonaise traditionnelle. En tant que père de triplés, il est à contre-courant dans la nouvelle société nippone obsédée par la surpopulation et où la famille traditionnelle est reléguée à un passé révolu. Son mariage confère un statut cosmopolite à ses triplés, «amérasiens» dont les noms « internationaux» (p.62), "Sonny, Sony et Soon-I» (p. 62), traduisent leur ethnie mêlée. La différence entre les prénoms est mise en évidence par les orthographes différentes qui soulignent les ascendances mixtes de chaque triplé. L'allusion à la marque "Sony », en référence à l'expansion économique du Japon moderne, met en valeur l'identité proprement japonaise du géniteur de l'enfant ainsi dénommé. « Sonny » évoque le mot anglais «sunny», signifiant "ensoleillé », et signale l'ascendance maternelle dominante. En effet, Mithra est d'essence solaire, à l'instar de la déesse mythique dont elle

\footnotetext{
${ }^{5}$ Il s'agit de cloisons translucides en papier.
} 
porte le nom, selon sa description par le catcher : «Ma femme, quand on la regarde, on a l'impression de voir le soleil. » (p. 61) "Soon-I » est un néologisme où le futur évoqué emblématise une Amérique cosmopolite, image d'un avenir gommant les identités pour les fusionner.

Les prénoms aux consonances anglaises des triplés s'intègrent à la langue hybride parlée dans le métro, où le métissage linguistique reflète celui des cultures. Les néologismes composites comme la «métrophobie » (1999b, p. 58), les «Euthanasiens», désignent les membres de la "société secrète d'entraide au suicide» (p.59). Les «lucinogènes» (p.60) sont les seuls médicaments autorisés aux Nocturnes, un allusion à la lumière refusée. Les croisements langagiers évoquent l'étouffement culturel qui mène les autochtones aliénés au suicide.

Le métro, à l'épicentre de l'affrontement entre le Japon traditionnel et la culture moderne venue de l'Occident, est un lieu de perdition. Il est assimilé au labyrinthe de la mythologie grecque, tandis que l'appropriation du mythe transforme le catcher en Thésée japonais. Sur le plan symbolique, le labyrinthe, «lieu de l'oubli et du musement » selon Bertrand Gervais, matérialise «l'errance provoquée par une multitude de choix à faire qui enfonce le sujet toujours plus profondément dans la confusion » (2008, p. 26 et 33). Le catcher tente de combiner les exigences de la tradition et celles de la vie moderne, mais il s'égare puis meurt. L'incipit de la nouvelle, «Les métros sont devenus des labyrinthes» (1999b, p. 57), vise tous les métros du monde, qui mènent leurs usagers à leur perte sous couvert de les transporter. La réunion de civilisations contraires sous l'effet du réalisme magique, dans la 
nouvelle, se solde par un échec existentiel pour quiconque tente d'assumer conjointement des modes de vie inconciliables.

\section{La bibliothèque, entre-deux réaliste magique}

Le second axe de notre étude concerne la pénétration d'un personnage à l'intérieur du texte, caractéristique des récits réalistes magiques. Il s'agit d'une technique visant à faire s'interpénétrer des mondes opposés grâce à l'immersion d'un personnage de lecteur dans le livre qu'il lit (Thiem, 1995, p. 235-238). Pour un lecteur de ce type, qui a l'impression d'être dans deux mondes à la fois, le livre est un refuge. Renonçant au détachement du simple lecteur, il devient luimême un acteur du monde livresque.

Dans « La petite princesse et le labyrinthe des rêves », les mondes opposés sont représentés par deux œuvres célèbres de la littérature enfantine, $A$ Little Princess de Frances Hodgson Burnett (1961 [1905]) et les Contes des mille et une nuits. L'héroïne du récit, Mira, est une petite métisse âgée de cinq ans, de père hindou et de mère d'origines britannique et hindoue. Orpheline de mère, Mira vit dans un petit village pauvre de l'Inde avec son oncle paternel. Son seul défaut, d'après ce dernier, est son « imagination trop vorace » (1999a, p. 29). Ne sachant pas encore lire, elle exige chaque soir que l'oncle lui raconte une nouvelle histoire. La nuit, elle appelle son père en pleurant. Un jour, ce dernier revient la chercher, et l'oncle est très malheureux. Des années plus tard, il reçoit des lettres exaltées de Mira, qui lui raconte son rêve d'étudier la bibliothéconomie et de partir à Londres travailler dans une 
grande bibliothèque dont ils parcourront ensemble « les allées remplies de livres merveilleux » (p. 37).

Le livre favori de Mira, $A$ Little Princess, au titre repris dans celui de la nouvelle, est porteur de l'idéologie de l'empire britannique. Elle est aussi passionnée par les Contes des mille et une nuits, l'œuvre la plus célèbre de la littérature orientale. Mira s'identifie autant à Sara Crewe, la jeune héroïne de Burnett, qu'à Schéhérazade, au point de se retrouver à égalité avec elles, au mépris de la logique et de la temporalité. Raconter des histoires est vital pour les trois fillettes qui recourent à l'imaginaire pour surmonter des situations très difficiles. Telle Sara Crewe, remédiant à sa déchéance en se racontant des histoires où elle est une princesse, et Schéhérazade obtenant la vie sauve grâce à ses histoires, Mira compense sa solitude en écoutant puis en imaginant des histoires. L'amour de l'oncle, pauvre mais instruit, pour les livres est égal à celui de sa nièce : la lecture est le «refuge contre [son] existence aliénante» (p. 29) de misérable ouvrier.

Une voix narrative tierce se fait entendre dans la nouvelle à travers Mira, anxieuse de sauvegarder son double patrimoine. L'imprégnation des cultures opposées s'effectue comme une digestion à laquelle fait allusion l'avidité de Mira et de Sara Crewe pour les histoires. Les fillettes sont littéralement assimilées à des rats de bibliothèque. Elles dévorent les livres, réincarnant les rats sacrés de la légende hindoue, d'après laquelle les rats «kabaas» (p. 30) sont les enfants de Karni Mata, sainte faiseuse de miracles ${ }^{6}$. La souris Melchisedec, alliée

\footnotetext{
${ }^{6}$ Selon la tradition, la déesse Karni Mata avait demandé qu'un enfant soigné par elle, appartenant à la caste des conteurs, revînt au monde sous l'apparence d'un rat.
} 
de Sara Crewe qui a passé sa petite enfance en Inde, se joint aux rats de la légende : " (dans certaines régions de l'Inde, les rats mangent à même les couverts des hommes, qui les considèrent comme les enfants de la déesse Karni Ma au même titre qu'eux, de sorte qu'on les appelle kabaas, ce qui signifie "enfants") » (p. 30).

La boulimie poussant Mira et Sara Crewe à réclamer puis à inventer toujours plus d'histoires introduit un cycle narratif hybride. La nouvelle, dont les protagonistes sont des enfants/rats ou des souris/enfants, associe la vieille croyance et la fiction anglaise. Par la valorisation de la croyance traditionnelle de l'Inde, les enfants défavorisés, rats réincarnés, s'épanouissent dans l'imaginaire.

On note également le syncrétisme par lequel la religion hindoue est unie à la tradition judéo-chrétienne à travers la figure de la souris Melchisedec, ainsi nommée par Sara Crewe d'après le prêtre-roi biblique, considéré comme le précurseur du Christ (McGillis, 1996, p. 79). Les références religieuses sacralisent la lecture rituelle de l'oncle hindou, le culte immémorial des rats sacrés confirmant la spiritualité de l’Inde dans sa pérennité.

Le greffage des merveilles du monde imaginaire de Sara Crewe sur les vieilles légendes de l'Inde a pour effet de niveler des imaginaires opposés. Un univers mental hybride se construit, issu de la contestation de l'idéologie impériale. Le métissage culturel de Mira est dû à sa double ascendance familiale, anglaise et hindoue. Petite-fille d'un officier britannique, elle est d'autant plus attirée par la culture anglophone qu'il s'agit d'ascendance maternelle. La mère disparue métaphorise la rupture du cordon ombilical avec le 
centre nourricier de l'empire, provoquant la perte d'identité des anciens colonisés. La nostalgie de Mira pour sa "mère » impériale se manifeste dans sa fascination pour la richesse matérielle : "Elle ne se lassait jamais d'entendre parler de ces villes modernes remplies de lumières, de voitures élégantes et d'hôtels luxueux» (1999a, p. 30). L'opposition des classes sociales recouvre celle des civilisations dans le contraste cruel entre les pauvres poupées de son offertes par l'oncle et les belles poupées anglaises de porcelaine apportées par le père. Ce dernier arrive " les bras chargés de cadeaux, tel un vrai Sahib", pour la petite fille qu'il appelle sa «maharani » (p. 35). Delhi, avec son prestige de grande métropole à l'occidentale, est aux antipodes du «village près de Rampur » (p. 28), anonyme et insignifiant.

La tendance anglophile de Mira est contrebalancée par son amour pour les livres de l'Inde et sa spiritualité latente, d'origine paternelle. Mira et l'oncle appartiennent au peuple hindou, détenteur d'une très riche tradition thaumaturgique, toujours vivante et agissante à travers leurs lectures qui les transportent littéralement, chaque nuit, dans l'univers merveilleux des légendes.

La théâtralisation est elle-même une mise dans le texte ludique par les deux acteurs, Mira et l'oncle, qui jouent au chat et à la souris, incarnant inlassablement "Schéhérazade et Shàhriyàr, Thésée, le Minotaure et Ariane » (1999a, p. 33). Les lecteurs d'histoires sont métamorphosés en acteurs, comme le montre le retour de la souris, furetant d'un registre culturel à un autre, telle Mira elle-même.

La quête de l'identité s'accompagne de déculturation. Le bazar hindou où l'oncle a trouvé $A$ Little Princess, par une 
appropriation de la culture d'origine étrangère, est le fonds inépuisable de l'imaginaire décrit comme une bibliothèque. À court d'histoires, forcé d'en inventer d'autres, «de [son] cru » (p. 30), comme Schéhérazade, l'oncle est lui-même, stimulé par Mira, l'auteur et le personnage de la nouvelle histoire.

- Je n'ai pas d'imagination ce soir...

- Alors, monte dans ta bibliothèque d'imagination.

- Il n'y a pas de livres! Tous les livres ont disparu!

- Dans ce cas, monte au 2e étage.

- Il y a un monstre qui me barre la route...

- Monte encore...

— L'escalier qui conduit au troisième a brûlé...

- Prends l'ascenseur.

(où a-t-elle été chercher ça?)

[...]

- Il y a un bandit qui a volé tous les livres pour enfants et qui se sauve par la fenêtre...

— Cours après lui. Vite! (p. 31-32)

L'élaboration ludique de l'aventure, « (Je frappe le bandit, paf paf paf, et il tombe comme un guignol)» (p. 32), accompagne l'invention d'un fonds d'histoires inédites, malgré le déni de Mira :

— JE VEUX MON HISTOIRE! [...]

- Mais je viens de t'en conter une...

- Ça ne compte pas. (p. 33).

La nouvelle histoire est issu du mélange de types littéraires, le Minotaure et le guignol, originaires de fonds culturels différents. L'oncle et la nièce, simultanément lecteurs, auteurs et acteurs, refondent le mythe ancien dans une version moderne conforme au style des bandes dessinées.

L'horreur bien naturelle de Mira pour les histoires sérieuses «pour adultes», destinées à « [faire] penser» (p. 31), 
ramène au registre de la littérature enfantine. Le lectorat enfantin, privilégié dans la nouvelle, exige une mise à niveau entraînant une distanciation par rapport au mythe. La saynète créée par Mira et son oncle subvertit la tragédie en spectacle drôle pour enfants. Dans la nouvelle, le comique l'emporte sur la tonalité grave de la tragédie grecque, dominante dans «Le catcher du métro ». Thésée, de pantin tragique, est devenu un héros de bande dessinée.

Le « labyrinthe des rêves » (1999a, p. 36), bibliothèque universelle où se trouvent toutes les histoires, est au carrefour des cultures. Comme chez Borges, la bibliothèque qui contient tous les livres est un labyrinthe. Telle Schéhérazade qui, représentée par Borges dans «Le jardin aux sentiers qui bifurquent", "se met à raconter textuellement l'histoire des 1001 Nuits, au risque d'arriver de nouveau à la nuit pendant laquelle elle la raconte » (Borges, 2002, p. 100), Mira, devenue adulte, en même temps qu'elle avance dans le dédale des rues de New Delhi, refait en sens inverse le chemin vers l'enfance. Évoluant simultanément vers l'avenir et le passé, elle réitère le récit de sa vie. La dynamique qui l'entraîne est celle-là même de l'écriture qui la mènera, "si tout marche bien », à la «vaste bibliothèque » de l'Université de Bombay, voire, «peut-être même, à Londres! » (1999a, p. 87) La projection dans le futur la ramène en dernier ressort à l'espace culturel du passé impérial, partie intégrante d'une histoire refaite à l'infini.

Les temps ont changé pour Sara et Mira, qui transgressent les conventions sociales d'une époque révolue. En racontant des histoires aux "enfants déguenillés » de la rue, Mira provoque « une petite révolution» (p.34) au village. Les «coins de rue» voués traditionnellement au troc, activité de survie économique, 
se transforment en "ashrams » (p. 34) et la conteuse devient le gourou des enfants défavorisés. Elle les soustrait à leur pauvreté en les faisant accéder à l'univers magique des contes, comme Sara Crewe lisant des contes de fées à ses condisciples émerveillées. Lorsque la snob Lavinia proteste contre la présence dans l'auditoire de Becky, la petite servante, Sara Crewe lui réplique que «les histoires sont à tout le monde» (Burnett, 1961, p. 47). Comme McGillis le constate, l'imaginaire est en mesure de "the power to fashion the self and the self's world ${ }^{7} »$. La littérature dite enfantine, politiquement marquée, n'est plus innocente. Raconter des histoires aux pauvres enfants hindous est un acte subversif qui restitue aux opprimés le statut qui leur a été usurpé dans le passé.

\section{Le poisson-écrivain}

Le pouvoir de l'écriture de réunir des mondes opposés dans une œuvre nouvelle apparaît également dans «Le royaume silencieux ", dont l'action se passe en Chine. Un poisson à visage humain, ressemblant à Mao Tsé Toung, a été pêché, déposé vivant dans un plat et presque mangé mais, sur le point d'être dépecé vivant puis consommé, il est jeté dans l'eau d'un vivier en dernière extrémité. Ses déplacements forcés du vivier au baquet au chaudron puis au plat font de lui un migrant. Sa famille a été tuée, sa mère harponnée et ses frères «aspirés brutalement vers le ciel»(1999c, p.146) par les pêcheurs. Depuis l'aquarium de l'école où on l'a plongé, il acquiert des notions de géographie utiles à ses futures pérégrinations de Chine en Alaska. Il finit par échouer sur une île en bordure du

\footnotetext{
7 « façonner le soi et le monde du soi » (1996, p. 70). Je traduis.
} 
pôle, où il se métamorphose en triton, divinité marine à corps d'homme et queue de poisson dans la mythologie grecque. Grâce au rallongement de ses nageoires, il peut taper à la machine. Loin des hommes cruels, accroché à son rocher arctique, le triton solitaire, mais heureux, s'adonne désormais à l'activité littéraire et lance ses manuscrits à la mer dans des bouteilles de whisky.

Le cycle de la métamorphose du poisson en écrivain est reproduit par les écrits voguant sur l'océan, figurant l'écrivain migrant, ballotté à l'infini entre les continents. Tel est le sort du poisson-écrivain, transplanté d'un milieu à l'autre, isolé, méprisé et incompris jusqu'à son accomplissement dans l'écriture. Dès sa naissance, sa différence avec ses congénères concerne le langage. Bien qu'incapable d'émettre des sons, le poisson s'exerce à « balbutier mentalement des mots » (p. 144), par une capacité qui le distingue de la masse des poissons muets. Sa différence morphologique interviendra plus tard. Déjà au stade indifférencié où il a l'air pareil aux autres poissons, il nourrit «des ambitions...métaphysiques » (p. 144), désireux de rompre le mutisme de ses congénères. Grâce à sa métamorphose, il réalise son désir d'expression dans l'écriture.

La mutation du poisson en triton apte à la création littéraire métaphorise la prise de parole par l'écrivain dans un monde où les masses soumises et silencieuses n'ont pas droit à l'expression. Sa monstruosité physique ultérieure ne fait que refléter celle, mentale, qui fait de lui un être différent et rejeté par tous, évoquant l'expérience de Chung lui-même. La similarité entre le poisson-écrivain et son auteur, trouvant tous deux leur identité dans la création littéraire, est accentuée par le contexte de leurs pratiques d'écriture respective. L'île du 
triton se réfère à Montréal, la ville-île peuplée d'immigrants destinés à demeurer des hybrides. Doté d'une capacité de survie hors pair et d'une grande faculté d'adaptation, le poisson-écrivain est le double de l'écrivain migrant.

Les deux règnes, animal et humain, auquel il appartient en même temps, comme les «lemmings humains » dans «Le catcher du métro » (1999b, p. 57-58), signalent la coexistence, en lui, de cultures divergentes. Ayant atteint le stade du triton, au terme de la métamorphose destinée à le faire survivre hors de l'eau, il se flatte d'appartenir « à ces créatures fabuleuses dans la mythologie» (1999b, p. 57-58). Pour le poisson autrefois sans contrôle sur sa vie, métaphorisant les peuples asiatiques colonisés et muselés dans le passé, la vraie vie est l'affirmation culturelle effectuée par l'appropriation du patrimoine des colonisateurs. La référence à la mythologie grecque renforce son statut dans l'île, où il règne "en monarque» (1999b, p. 57-58). Prenant sa revanche sur l'impérialisme occidental, le poisson-écrivain s'élève au rang d'un demi-dieu marin, lorsqu'il récupère de surcroît, sur l'épave du bateau échoué, les instruments conventionnels de l'écriture, la machine à écrire et le whisky, adjuvant à la création littéraire.

Il y avait aussi des livres dont les pages à moitié effacées par l'eau formaient un curieux mélange de silence et de mots rescapés. Tout à coup, la pureté des syllabes liquides constituant mon environnement sonore me parut décevante et j'éprouvai une vive nostalgie pour ce qui n'était pas la plénitude du sable, de l'air et de l'eau. C'est alors que, sortant du silence où je m'étais enseveli, je découvris de nouveau la nécessité des mots. (1999c, p. 152)

Construisant son identité sur les ruines de l'Occident naufragé, le poisson métaphorise l'écrivain migrant qui érige 
son univers propre en recyclant les débris de cultures en perdition. Le poisson-écrivain est un survivant échappé à l'inexistence des inférieurs muets en "sortant du silence où [il s'était] enseveli » (1999c, p. 155). Mourir ou écrire, tel est le dilemme de l'ancien dominé, forcé, pour survivre, de créer une langue et une culture nouvelles.

\section{Un autre monstre: l'écrivain jeune-vieux}

On assiste à une autre représentation de l'écrivain en monstre hybride dans « Le baiser de minuit ». Le protagoniste de l'action est un jeune islandais atteint de progeria, maladie génétique rare provoquant des changements physiques faisant ressembler ceux qui en sont atteints à des vieillards. Il raconte l'histoire de sa vie sur le mode autodiégétique. La maladie l'a fait déchoir, à l'âge d'un an, de son titre de «plus beau bébé d'Islande» (p. 38). L'accélération anormale du métabolisme, causée par la maladie, s'accompagne chez lui d'un développement intellectuel exceptionnel. Très jeune, il est passionné de lectures médicales, elles-mêmes monstrueuses, produites par «des mots longs et terrifiants comme des scolopendres» (p.39). La monstruosité produite par l'interaction du lecteur et de l'écriture transforme l'écrit étalé sur la page en mille-pattes carnivore et venimeux, doté d'un pouvoir propre. Du poisson-écrivain on passe aux mots-reptiles lus et écrits par le malade. Ce dernier, nourrissant la double ambition de devenir écrivain et médecin, est à la fois le sujet et l'objet de recherches médicales approfondies.

Son amie Nuit-Blanche est une petite Japonaise albinos allergique au soleil, amenée en Islande par la «maladie 
déroutante » (p. 34) qui, littéralement, la mène en Islande en la faisant dévier de son itinéraire de vie normal. À l'hôpital, la fête de Noël finit mal, car le garçon au visage repoussant et sa belle amie ont été contraints d'échanger un baiser sans que les adultes s'y opposent. Contrairement à Nuit-Blanche, dont il lit le dégoût sur son beau visage, le baiser procure au garçon un bonheur ineffable.

Comme Mira, au visage prématurément ridé par les nuits de lecture, le garçon, à la fois jeune et vieux, trouve dans l'écriture le remède à la mort et à l'anonymat. On apprend dès l'incipit qu'il n'a pas d'identité propre. «Mon nom n'a aucune importance. Les gens qui vivent quelques années seulement n'ont pas besoin de nom » (2003a, p. 27). Il subit le même sort que le petit village déshérité de l'Inde dans « La petite princesse et le labyrinthe des rêves ». Son seul recours, pour exister en tant qu'individu, est d'écrire le récit de sa vie.

Les éléments narratifs sont des doublets antinomiques, tels l'enfant adulte, la petite Japonaise blanche, le soleil et la nuit. La célébration de Noël à l'hôpital, avec ses rites particuliers, unit paradoxalement la joie et la douleur. L'hôpital est le lieu privilégié de la monstruosité due à la maladie métaphorisant, selon Eloïse Brézault, « un métissage impossible entre deux mondes » qui « prend tout son sens lorsque l'auteur met en scène des couples mixtes dont l'union ne peut durer comme si ces couples portaient, en eux, une malédiction » (2001). L'étreinte forcée du jeune garçon très vieux et laid et de la petite japonaise blanche et très belle est un «baiser au lépreux ${ }^{8}$ » (2003a, p. 38). La référence au Christ qui, par amour

\footnotetext{
${ }^{8}$ Le Baiser au lépreux (1922) est le titre d'un roman de F. Mauriac.
} 
des hommes, se sacrifie pour les sauver, est incluse dans l'union monstrueuse de l'Orient et l'Occident, réduplication du métissage des religions chez le garçon, davantage bouddhiste que chrétien. En effet, s'étant abstenu de sommeil trois jours durant, il s'identifie aux «bonzes marathoniens » devenus des « divinités vivantes » (p. 33).

Il a des "pensées doubles » l'apparentant aux héros de Dostoïevski, tout en rejouant des scènes des Mille et une nuits avec la petite Japonaise: "[...] c'est la nuit que la magie commence: elle sort de sa chambre comme un génie de sa lampe d'Aladin, et moi je suis le prince persan qui la conduit à travers les mille et une nuits de notre royaume. » (p. 35)

Les Mille et une nuits participent du même brassage littéraire que l'écrit hybride dans «La Petite princesse et le labyrinthe des rêves », où il est la seule parade à la disparition et à la mort. Aussi bien, le journal intime du jeune Islandais est son testament, car sa vraie vie est l'écriture, «trace de [son] bref séjour sur terre » (2003a, p. 35), par laquelle il remédie à son altérité monstrueuse. Comme le montre Wendy B. Faris, Shéhérazade représente l'écrivain menacé de mort dans la modernité (1995, p.163-190). Pour les enfants hybrides, comme pour le poisson-écrivain, la création littéraire est le seul moyen de s'affirmer dans un monde qui les condamne à l'inexistence. L'acte d'écriture du jeune Islandais prend la valeur d'une nouvelle naissance malgré la mort prochaine et inéluctable. L'écrit nouveau, dans son hybridité même, s'approprie les mythes de renaissance'.

\footnotetext{
${ }^{9}$ Le titre de la nouvelle, « Le baiser de minuit » est une référence aux Enfants de Minuit de Salman Rushdie (1983) où, symboliquement, la naissance des enfants coïncide avec celle de l'Inde nouvelle. La monstruosité du jeune
} 


\section{Le réalisme magique en noir et blanc}

Nous consacrerons la dernière partie de notre étude à un bref examen de "L'enfantement». Le rattachement explicite de la nouvelle au conte oral est explicite, placé en exergue : «Inspiré d'un conte coréen » (2003b, p. 139). L'incipit, «Il était une fois » (p.139), confirme l'appartenance de la nouvelle au genre. Évoquant Blanche-Neige, figure mythique d'origine germanique très populaire en Europe et en Amérique du Nord, la protagoniste du récit, Blanche, est une petite Coréenne à la peau très pâle, surnommée «la fille de neige » (p. 139), chassée de son village natal parce que sa blancheur fait peur ${ }^{10}$. Une chamane l'emmène dans une forêt magique. Par une nuit de tempête, un homme la possède sans mot dire. Blanche met au monde un enfant glacé à la peau bleue et transparente qui fond parce qu'il s'est trop approché $\mathrm{du}$ feu. Les cheveux de Blanche sont devenus noirs. De retour au village où on la traite en reine, elle repart cependant dans la forêt et retrouve son enfant de glace.

La communauté rurale superstitieuse, en retrait du monde, caractérise l'univers réaliste magique, situé à la campagne plutôt qu'à la ville (Faris, 1995, p.182), où «le quotidien est souvent bouleversé par un événement anormal, bizarre, étrange, magique» (Vautier, 1991). Blanche et son enfant, rejetés par les villageois, sont des migrants et, comme tels, porteurs d'une altérité considérée comme anormale par les autochtones. Toutefois, son second départ, volontaire, de son village natal montre qu'elle assume sa différence. Non

islandais évoque la difformité de Saleem, le héros-narrateur du roman, métaphorisant l'Histoire, « malade», de son pays.

${ }^{10}$ Blanche est une réduplication de Nuit-Blanche, la petite japonaise albinos. 
seulement elle fait sien un système de valeurs où la chevelure noire ne dénote pas la conformité au modèle dominant, mais encore son départ définitif frappe la communauté de stérilité, marquant la fin de la civilisation primitive.

L'élément magique moteur du conte est la blancheur, comme dans Les Aventures d'Arthur Gordon Pym (1994 [1837]) d'Allan Edgar Poe où, selon Borges, le blanc est assimilé à la tempête et à la mort dans les vieilles croyances des autochtones à la peau noire (1981 [1932], p. 78). Le réalisme magique revêt une dimension critique dans la réécriture du conte de fées, où l'opposition noir/blanc est retournée. À son retour au village, Blanche a les cheveux noirs mais ceux des habitants sont «blancs comme neige » (2003b, p. 154) et ils ne peuvent plus avoir d'enfants depuis son bannissement. Comme précédemment, le renouveau s'accomplit dans la forêt, espace menaçant mais finalement accueillant où, à son retour, "l'enfant de glace [fait] un salut du bras, chac-chac, chac » (p. 155) dans son langage à lui. La renaissance s'accompagne de l'expression inédite du sens nouveau de la vie dans un présent rejetant le joug des coutumes et des croyances anciennes.

Michel Dupuis et Albert Mingelgrün constatent que les enfants, dans bon nombre de fictions, sont dotés de «leurs dispositions ludiques, leur imagination, leur promptitude à apprendre», qui les prédestinent «à percevoir les indices magiques» (1987, p. 230). Cependant, dans les nouvelles de Chung, les enfants sont orphelins ou ont été abandonnés par leurs parents. Chez eux, la perte du père ou de la mère métaphorise le déracinement des peuples colonisés et infantilisés. Démunis et impuissants, ils n'ont guère d'autre choix que l'écriture pour trouver leur identité. Grâce au réalisme magique, ils actualisent 
leurs potentialités créatrices en élaborant un langage nouveau, porteur de significations nouvelles.

\section{Conclusion}

En conclusion, après avoir d'abord établi le lien entre le réalisme magique, le discours post-colonialiste et l'écriture migrante, dont les auteurs sont souvent originaires de pays non occidentaux, nous avons vu que la stratégie d'écriture réaliste magique traduit la condition des immigrés vivant «entre des mondes", selon l'expression de Iain Chambers ${ }^{11}$. Dans un second temps, nous avons envisagé le réalisme magique, dans les nouvelles de Chung, comme technique littéraire postcoloniale, promotrice d'une écriture inédite exhibant ses diverses origines refondues dans des formes éclectiques. Appelé à donner le droit à l'expression libre aux anciens dominés, le surnaturel, aux marges de la rationalité, s'avère apte à assurer leur survie et leur affirmation. Au troisième stade de notre étude, nous avons constaté que, contrairement au catcher qui se suicide, à l'instar de nombre de ses congénères nocturnes, perdus comme dans un labyrinthe menant à une impasse, les protagonistes des autres récits de Chung trouvent dans l'écrit, que ce soit le leur en propre ou tous les livres, l'alternative possible à l'ambiguïté. Nous avons vu que la figure de Schéhérazade sert de modèle au conteur échappant à la mort. De la même façon que les histoires de Schéhérazade incluent la sienne propre, la bibliothèque, reproduite par le dédale des rues de New Delhi, à l'image du métro labyrinthique

11 « living between worlds » $(1994$, p. 27). Je traduis. 
de Tokyo circulant entre les civilisations, contient toutes les histoires que racontent les personnages eux-mêmes, telle, dans la "grande ville», Mira, devenue «une grande personne» (1999a, p. 36-37) qui cherche sa voie propre, métaphorisant la quête d'identité culturelle de l'Inde. Le réalisme magique a transformé les rues et le métro des grandes métropoles modernisées de l'Orient en domaine exclusif de l'imaginaire. L'absorption du substrat légendaire ancien dans la narration nouvelle, présentifiée par la non moindre fascination de Mira pour la littérature de l'Inde traditionnelle que pour la culture anglaise, assure sa survie à travers la voix qui s'immisce pour la faire perdurer. La bipolarité des espaces et des temps fonde un récit d'essence onirique magique qui s'auto-raconte comme une équipée dans un labyrinthe.

En définitive, dans les nouvelles que nous avons examinées, la coprésence de la normalité et du surnaturel implique la superposition des cultures et des traditions, caractéristique de l'écriture migrante qui, selon Nepveu, intronise « un mouvement culturel pour lequel [...] le métissage, l'hybridation, le pluriel, le déracinement sont des modes privilégiés »(1988, p. 201). Par leur recours aux techniques narratives réalistes magiques, les nouvelles de Chung s'insèrent dans une postmodernité appelée à traduire l'essence duelle de l'identité et de l'histoire ${ }^{12}$.

12 Voir à ce sujet Linda Hutcheon (1989, p. 154). 


\section{Bibliographie}

BAKER, Suzanne. (1993), « Binarisms and Duality: Magic Realism and Postcolonialism », SPAN: Journal of the South Pacific Association for Commonwealth Literature and Language Studies, $\mathrm{n}^{\circ} 36$, p. 82-87.

BERNIER, Silvie. (2002), Les Héritiers d'Ulysse, Outremont, Lanctôt.

BoRGES, Jorge Luis. (1981 [1932]), « Narrative Art and Magic » (1932), dans Emir RodRiguez Monegal et Alastair ReId (dir.), Borges, a Reader: A Selection from the Writings of Jorge Luis Borges, trad. R. Simms, New York, Dutton, p. 34-38.

—. (2002 [1941]), «Le jardin aux sentiers qui bifurquent», Fictions, trad. Paul Verdevoye, Nestor Ibarra et Roger Caillois, Paris, Gallimard, p. 91-104.

BRÉZAULT, Éloïse. (2001), «Démystification et mal-être dans le roman contemporain en Afrique Noire», La Question des savoirs (Notre Librairie), $\mathrm{n}^{\circ} 144$, <http://www.culturesfrance.com/librairie/derniers/144/som m144.htm>.

BuRnetT, Frances Hodgson. (1961 [1905]), A Little Princess, Londres, Penguin.

ChAmbers, Iain. (1994), Migrancy, Culture, Identity, Londres, Routledge.

Chanady, Amaryll Beatrice. (1985), Magical Realism and the Fantastic: Resolved versus Unresolved Antinomy, New York, Garland. 
-. (2003), "Rereading Quebecois Literature in a Postcolonial Context », Quebec Studies, n 35, p. 31-34.

Chung, Ook. (1999 [1994]), Nouvelles orientales et désorientées, Paris, Le Serpent à Plumes.

—. (2003), Contes butô, Montréal, Boréal.

DuPuIS, Gilles. (2005), «L'Orient désorienté. Le topos du Chinatown dans quatre romans contemporains ", Voix et Images, vol. 31, n 1, p. 101-114.

DuPUIS, Michel et Albert MingelgrÜN. (1987), "Pour une poétique du réalisme magique », dans Jean Weisberger (dir.), Le Réalisme magique, roman, peinture, cinéma, Lausanne, L'Âge d'Homme, p. 219-232.

FARIS, Wendy B. (1995), "Scheherazade's Children: Magical Realism and Postmodern Fiction», dans Lois PARKINSON ZAMORA et Wendy B. FARIS (dir.), Magical Realism: Theory, History, Community, Durham et Londres, Duke UP, p.163-190.

GERVAIS, Bertrand. (2008), La Ligne brisée. Labyrinthe, oubli et violence. Logiques de l'imaginaire. Tome II, Montréal, Le Quartanier.

HutChEON, Linda. (1989), "Circling the Downspout of Empire: Post-Colonialism and Postmodernism », ARIEL, vol. 20, $\mathrm{n}^{\circ} 4$, p. 149-175.

MAURIAC, François. (1922), Le Baiser au lépreux, Paris, Grasset. McGILLIS, Roderick. (1996), A Little Princess. Gender and Empire, New York, Twayne.

NePVEU, Pierre. (1988), L'Écologie du réel, Montréal, Boréal. 
PoE, Edgar Allan Poe. (1994 [1837]), The Narrative of Arthur Gordon Pym, Oxford, Oxford UP.

RuSHDIE, Salman. (1983 [1981]), Les Enfants de minuit, trad. Jean Guiloineau, Paris, Stock.

SLEmon, Stephen. (1988), «Magic Realism as Post-Colonial Discourse », Canadian Literature, n 116 , p. 9-24.

SugARS, Cynthia. (2002), « National Posts: Theorizing Canadian Postcolonialism ", International Journal of Canadian Studies/ Revue internationale d'études canadiennes, $\mathrm{n}^{\circ} 25$, p. 15-41.

ThiEM, Jon. (1995), « The Textualization of the Reader in Magical Realist Fiction », dans Lois PARKINSON ZAMORA et Wendy B. FARIS (dir.), Magical Realism, Durham et Londre, Duke UP, p. 235-238.

Tiffin, Helen. (1988), « Post-Colonialism, Post-Modernism, and the Rehabilitation of Post-Colonial History ", Journal of Commonwealth Literature, vol. 23, n 1, p. 169-181.

VAUTIER, Marie. (1991), «La révision postcoloniale de l'histoire et l'exemple réaliste magique de François Barcelo », Études en littérature canadienne, vol. 16, $\mathrm{n}^{\circ} 2$. $<$ http://www.lib.unb.ca/Texts/SCL/bin/cgi? directory=vol16_2/\&filename=Vautier.htm $>$.

—. (2003), "Les pays du nouveau monde, le postcolonialisme de consensus, et le catholicisme québécois », Québec Studies, $\mathrm{n}^{\circ} 35 . \quad<$ http://find.galegroup.com/itx/infomark.do?\& contentSet $=\mathrm{IAC}>$. 


\title{
Résumé
}

Simone Grossman analyse des textes de deux recueils, Nouvelles orientales et désorientées (1994) et Contes butô (2003) d'Ook Chung, sous l'optique du réalisme magique. Elle montre combien ce genre se prête à l'expression de l'expérience migrante, tout en faisant appel à la théorie postcoloniale. Dans un premier temps, la critique examine comment le réalisme magique catalyse l'interpénétration critique des cultures. Puis, elle s'intéresse au topos réaliste magique de la lecture et de la bibliothèque. Enfin, l'étude traite des personnages d'enfants qui, par-delà leurs dispositions extraordinaires, métaphorisent les colonisés.

\begin{abstract}
Simone Grossman analyses texts from to short story collections of Ook Chung, Nouvelles orientales et désorientées (1994) and Contes butô (2003), through the lens of magic realism. She demonstrates how this genre lends itself to the expression of the migrant experience, invoking as well postcolonial theory. First, she examines how magical realism catalyzes the critical interpenetration of cultures. Then, she looks at the magical realist tropes of reading and the library. Finally, the study deals with child characters who, beyond their extraordinary traits, serve as metaphors of the colonized.
\end{abstract}

